\title{
Clinical Significance of Molecular Biomarkers on Brain Gliomas
}

\author{
Shanqiang $\mathrm{Qu}^{1 *}$, Shuting $\mathrm{Li}^{2}$ and Zhicheng $\mathrm{Hu}^{3}$ \\ ${ }^{1}$ Department of Neurosurgery, the First Affiliated Hospital, Sun Yat-sen University, Guangzhou 510080, China \\ ${ }^{2}$ Department of Plastic surgery, the First Affiliated Hospital, Sun Yat-sen University, Guangzhou 510080, China \\ ${ }^{3}$ Department of Burn Surgery, the First Affiliated Hospital, Sun Yat-sen University, Guangzhou 510080, China
}

*Corresponding author: Shanqiang Qu, Department of Neurosurgery, the First Affiliated Hospital, Sun Yat-sen University, Guangzhou 510080, China.

Received Date: August 20, 2020

Published Date: August 31, 2020

\section{Mini Review}

Gliomas are the most common primary brain tumors, and more than half of them are glioblastoma multiformes (GBMs) with the highest heterogeneity malignant degree. According to the statistical report of the Central Brain Tumor Registry of the United States, the glioma patients in the United States accounted for $26 \%$ of all intracranial tumor patients and $81 \%$ of all intracranial malignant tumor patients in 2011-2015 [1]. The overall survival (OS) of patients with gliomas has always been

nsatisfactory. Although the patients with GBMs underwent standardized treatment, the median OS of them is only about 12 months [2]. Therefore, it is very important to further clarify the pathogenesis of glioma, find new targets and put forward new diagnostic and prognostic biomarkers. Some patients with the same pathological diagnosis have great differences in the OS, which indicates the deficiency of traditional pathological indicators in judging the prognosis of patients. In recent years, great progress has been made in the study of molecular biomarkers which are helpful to the clinical diagnosis and prognosis of gliomas have been found. To further understand the molecular biological characteristics of glioma and identify more potential molecular markers is of great significance for revealing the pathological mechanism and clinical diagnosis and prognosis of glioma.

\section{Isocitrate dehydrogenase (IDH) mutation}

IDH is an important rate-limiting enzyme in tricarboxylic acid cycle, which could be of guiding significance to the diagnosis and prognostic prediction of gliomas. IDH mutation often occurs in WHO grade II-III gliomas, such as diffuse astrocytoma, oligodendroglioma and secondary glioblastoma [3]. Among them, IDH1 mutation is more common in diffuse astrocytoma and IDH2 mutation is more common in oligodendroglioma [4]. In addition, IDH mutation has the good predictive value for the prognosis of high-grade gliomas, but the prognosis of low-grade diffuse gliomas is not clear [5]. IDH mutant enzyme inhibitors are also a good direction of clinical drug research in the future.

\section{1p/19q loss of heterozygosity}

Chromosomal $1 \mathrm{p} / 19 \mathrm{q}$ loss of heterozygosity occurs in oligodendroglioma and anaplastic oligodendroglioma [6]. Therefore, $1 \mathrm{p} / 19 \mathrm{q}$ co-deletion is considered to be a reliable molecular marker for the diagnosis of oligodendroglioma [7]. In addition, previous studies have shown that $1 \mathrm{p} / 19 \mathrm{q}$ co-deletion is also a favorable protective factor for the prognosis of glioma patients. Notably, increasing studies showed Oligodendroglioma patients with $1 \mathrm{p} / 19 \mathrm{q}$ co-deletion are much more sensitive to chemotherapy [8]. The mechanism is likely to be due to epigenetic changes induced by metabolism.

\section{MGMT promoter methylation}

The incidence of MGMT promoter methylation was 60\% $80 \%$ in oligodendrogliomas and $20 \%-45 \%$ in GBMs. Increasing studies showed that glioma patients with MGMT promoter methylation are more sensitive to chemotherapy and have longer OS [9]. Therefore, MGMT promoter methylation is usually used as a sensitivity indicator for chemotherapy. However, some patients 
with MGMT promoter methylation may show pseudo-progression after receiving chemotherapy, which should raise the clinicians attention.

\section{BRAF abnormality}

BRAF is a member of RAS / RAF / MEK / ERK pathway, which plays an important role in regulating cell growth, proliferation and survival. The abnormality of BRAF gene in central nervous system mainly include the fusion of BRAF V600E mutation and BRAF [10]. BRAF fusion mainly occurs in capillary stellate cell tumor and diffuse leptomeningeal glial neuronal tumor. BRAF V600E mutations mainly occur in IDH wild-type gliomas, including pilocytic astrocytoma, gangliocytic glioma, anaplastic ganglioglioma and pleomorphic xanthoastrocytoma [11]. About $80 \%$ of pilocytic astrocytoma contain KIAA 1549-BRAF gene fusion, and $10 \%$ of children with low-grade gliomas have BRAFV600E mutations.

\section{ATRX mutation}

ATRX mutation is common in WHO grade II-III astrocytomas and secondary glioblastomas, but rarely in primary glioblastomas, rare in oligodendroglioma, which could be regarded as a molecular phenotypic marker of astrocytoma [12]. However, the impact of loss of ATRX expression on the survival and prognosis of glioma patients is still controversial [13].

\section{PTEN mutation}

As a tumor suppressor gene, PTEN inhibits tumor proliferation by blocking cell division cycle. PTEN gene mutation is most common in patients with GBMs. Mellinghoff et.al reported that the coexpression of PTEN and EGFRVIII can increase the sensitivity of glioma to erlotinib, but the loss of PTEN will lead to drug resistance [14].

\section{TERT promoter mutation}

TERT promoter mutation is common in oligodendrogliomas and primary GBMs. TERT promoter mutation with IDH wild-type is the most common genotype of primary GBMs. The poor prognosis of patients with IDH wildtype is closely related to TERT mutation [15]. However, TERT mutation is a favorable prognostic factor in IDH mutation glioma [16].

\section{Conclusion}

Glioma is still a kind of tumor with poor prognosis among systemic tumors. However, with the development of molecular biology technology, the discovery of many molecular biomarkers provides an important reference for the accurate diagnosis, prognosis and individualized treatment of gliomas. IDH mutation is a favorable factor for patients' prognosis. IDH mutation is often accompanied by MGMT promoter methylation in glioma patients, which often indicates that patients are sensitive to TMZ chemotherapy. The $1 \mathrm{p} / 19 \mathrm{q}$ co-deletion is a favorable factor for prognosis of patients. In addition, it is also a reliable diagnostic marker for oligodendroglioma. At the same time, the $1 p / 19 q$ codeletion is also a predictor of the effectiveness of TMZ treatment. TERT promoter mutation was a favorable prognostic factor in IDH mutated tumors, whereas it was an unfavorable prognostic factor in IDH wildtype tumors. BRAF fusion is a reliable evidence of pilocytic astrocytoma. The mutation of BRAF V600E is more complicated. BRAF V600E mutations suggest that patients may be sensitive to BRAF inhibitors, such as vemurafenib.

\section{Acknowledgement}

None.

\section{Conflict of Interest}

No conflict of interest.

\section{References}

1. Ostrom QT, Gittleman H, Truitt G, Boscia A, Kruchko C, et al. (2018) CBTRUS Statistical Report: Primary Brain and Other Central Nervous System Tumors Diagnosed in the United States in 2011-2015. Neuro Oncol 20: iv1-iv86.

2. Witthayanuwat S, Pesee M, Supaadirek C, Supakalin N Thamronganantasakul K, et al. (2018) Survival Analysis of Glioblastoma Multiforme. Asian Pac J Cancer Prev 19: 2613-2617.

3. Liu S, Shah Z, Sav A, Russo C, Berkovsky S, et al. (2020) Isocitrate dehydrogenase (IDH) status prediction in histopathology images of gliomas using deep learning. Sci Rep 10: 7733.

4. Hartmann C, Meyer J, Balss J, Capper D, Mueller W, et al. (2009) Type and frequency of IDH1 and IDH2 mutations are related to astrocytic and oligodendroglial differentiation and age: a study of 1,010 diffuse gliomas. Acta Neuropathol 118: 469-474.

5. Gozé C, Blonski M, Le Maistre G, Bauchet L, Dezamis E, et al. (2014) Imaging growth and isocitrate dehydrogenase 1 mutation are independent predictors for diffuse low-grade gliomas. Neuro Oncol 16: 1100-1109.

6. Li J, Miao N, Liu M, Cui W, Liu X, et al. (2014) Clinical significance of chromosome $1 \mathrm{p} / 19 \mathrm{q}$ loss of heterozygosity and Sox17 expression in oligodendrogliomas. Int J Clin Exp Pathol 7: 8609-8615.

7. Louis DN, Perry A, Reifenberger G, von Deimling A, Figarella-Branger D, et al. (2016) The 2016 World Health Organization Classification of Tumors of the Central Nervous System: a summary. Acta Neuropathol 131: 803-820.

8. Cairncross JG, Ueki K, Zlatescu MC, Lisle DK, Finkelstein DM, et al. (1998) Specific genetic predictors of chemotherapeutic response and survival in patients with anaplastic oligodendrogliomas. J Natl Cancer Inst 90: 1473-1479.

9. Weller M, Stupp R, Reifenberger G, Brandes AA, van den Bent MJ, et al. (2010) MGMT promoter methylation in malignant gliomas: ready for personalized medicine? Nat Rev Neurol 6: 39-51.

10. Jones DT, Kocialkowski S, Liu L, Pearson DM, Bäcklund LM, et al. (2008) Tandem duplication producing a novel oncogenic BRAF fusion gene defines the majority of pilocytic astrocytomas. Cancer Res 68: 86738677.

11. Schindler G, Capper D, Meyer J, Janzarik W, Omran H, et al. (2011) Analysis of BRAF V600E mutation in 1,320 nervous system tumors reveals high mutation frequencies in pleomorphic xanthoastrocytoma, ganglioglioma and extra-cerebellar pilocytic astrocytoma. Acta Neuropathol 121: 397-405.

12. Jiao Y, Killela PJ, Reitman ZJ, Rasheed AB, Heaphy CM, et al. (2012) Frequent ATRX, CIC, FUBP1 and IDH1 mutations refine the classification of malignant gliomas. Oncotarget 3: 709-722. 
13. Ebrahimi A, Skardelly M, Bonzheim I, Ott I, Mühleisen H, et al. (2016) ATRX immunostaining predicts IDH and H3F3A status in gliomas. Acta Neuropathol Commun 4:60.

14. Mellinghoff IK, Wang MY, Vivanco I, Haas-Kogan DA, Zhu S, et al. (2005) Molecular determinants of the response of glioblastomas to EGFR kinase inhibitors. N Engl J Med 353: 2012-2024.

15. Killela PJ, Reitman ZJ, Jiao Y, Bettegowda C, Agrawal N, et al. (2013) TERT promoter mutations occur frequently in gliomas and a subset of tumors derived from cells with low rates of self-renewal. Proc Natl Acad Sci U S A 110: 6021-6026.

16. Arita H, Yamasaki K, Matsushita Y, Nakamura T, Shimokawa A, et al. (2016) A combination of TERT promoter mutation and MGMT methylation status predicts clinically relevant subgroups of newly diagnosed glioblastomas. Acta Neuropathol Commun 4: 79. 